\title{
Photovoltaic Inverters with Fault Ride-Through Capability
}

\author{
Gustavo M. S. Azevedo \\ Electrical Department of Engineering and Power Systems \\ Federal University of Pernambuco (UFPE) \\ Recife, Pernambuco Brazil \\ Contact Author: gustavomsa@aim.com
}

\author{
Gerardo Vazquez, Alvaro Luna, Daniel Aguilar, \\ Alejandro Rolan \\ Electrical Department of Engineering \\ Technical University of Catalonia \\ Colom 1- 08222-Terrassa, Spain
}

\begin{abstract}
This paper presents a new control strategy that allow the photovoltaic system operate under grid faults without overpass the rated current and assuring sinusoidal currents. In the classic control strategies used in photovoltaic systems the power delivered to the grid remains constant when a fault occurs, hence the current can reach dangerous values. Therefore the converter has a protection system to disconnect it avoiding its damage. Thus it must be connected manually when the electrical grid is re-established. The strategy presented here overcomes these drawbacks. It is compared with the classic strategy through simulations in PSCAD and the experimental results prove its effectiveness.
\end{abstract}

\section{INTRODUCTION}

In nowadays the overall power system is changing, a large number of Distributed Generation (DG) units, such as wind turbines, Photovoltaic (PV) generators, fuel cells, are being developed and installed. In several countries the PV power supplied to the electrical grid is gaining more and more visibility due to many governmental incentives. The development and growth of grid-connected systems is demanding a better evaluation of the performance of the available PV systems under fault conditions, as well as the quality of the energy generated by them.

There are a lot of power converter topologies for PV systems such as two-stage, single-stage, with transformer, transformerless and others. However all these topologies require an inverter to connect them to the grid and several requirements must be met to perform this connection. One of these requirements is about the under voltage protection. The Germany standard DIN/VDE 0126 [1], for example, says that overvoltage upper to $15 \%$ and undervoltage lower than $20 \%$ must lead to the disconnection from the grid within $200 \mathrm{~ms}$. On the other hand the philosophy of disconnecting DG from the system "at first sign of trouble" might not be the optimal approach. Repeated disconnections may have a negative impact on components' lifetime as well as causing further disturbances on the grid [2]. Thus the lifetime and reliability of whole system can be improved if the inverter was able to keep connected as long as possible. But none of the commercial PV inverters tested in [2] was able to do this.

This paper shows that the actual control strategies used in the PV systems cause harmonic current injections on the grid and dangerous overcurrents when voltage sags occurs and trip protections are necessary to avoid the PV inverter damage. The paper also proposes a new control strategy to overcome these drawbacks and permit the inverter operate under any unbalanced condition. The control is made on stationary reference frame using Proportional-Resonant (PR) current control [3]. The DC link voltage is controlled through the active power delivered by the PV system. Instead of using a hard saturation on reference currents, the control calculates dynamically the maximum active power that the inverter can deliver and use this value as limiter to the reference power. The current references are calculated based on the positivesequence voltage in such way to result in balanced values even in presence of unbalanced grid voltages. The new strategy present here is evaluated through simulations in PSCAD/EMTDC and the experimental results prove its effectiveness.

\section{PV SYSTEM CONTROL}

The Fig. 1 shows a generic diagram of a single-stage photovoltaic system. Basically it is composed of a PV array, a Voltage Source Inverter (VSI), a LC output filter to connect the VSI to the electrical grid and the control system. The fist control block is a Maximum Power Point Tracking (MPPT) used to set the point of PV array operation is such way to maximize its power generation [3]. The MPPT output is the DC voltage reference used by the DC link voltage controller. The controller of the DC link voltage can act on the direct current, in the controllers based on synchronous reference frame [4][5], or on the active power, in the controllers based on stationary reference frame [6].

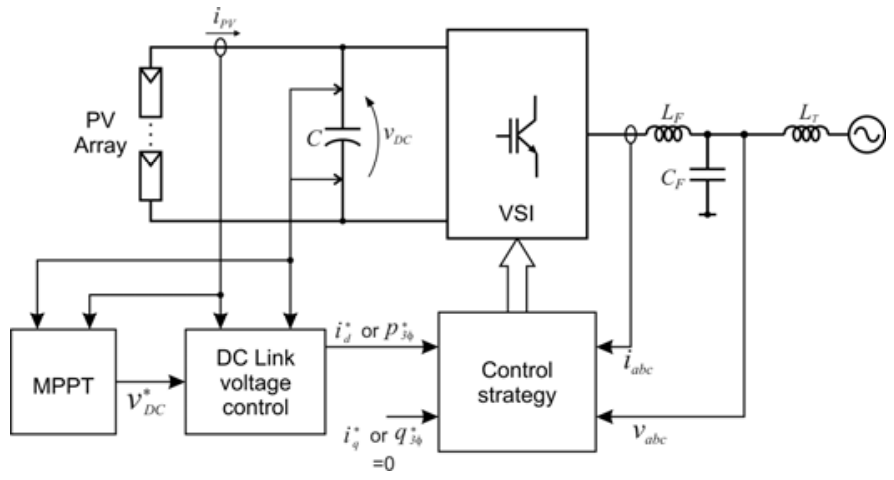

Fig. 1.Diagram of the photovoltaic system 
Generally the PV system control is designed considering only the balanced and sinusoidal condition which results in errors under fault situations. These errors can appear due to PLL synchronization system and the oscillation on Proportional Integral (PI) controllers references. However there are some ways to overcome these problems as enhanced PLL strategies, current controls in a double synchronous reference frame or using PR controllers and others.

On the other hand the main drawback of these controls is the overcurrent that occurs due to grid unbalanced voltage. The DC link voltage control is generally designed to keep the DC voltage equal to the reference in any way. It means that the active power is always constant and it results in distorted currents when the voltage is unbalanced. The Fig. 2 shows an example of what happen with the PV system (using the classic control strategy) when an asymmetrical fault occurs. More information about the simulation conditions will be described in the next section. In this example the overcurrent was 51\% and the currents are completely distorted throughout the fault. Note that both DC voltage and active power are constant at any time as mentioned before. An improvement can be obtained if the DC voltage controller is designed to allow oscillations on DC voltage. The overcurrent, in this case, fell down to $22 \%$ with this change as well the currents became sinusoidal. However a better result can be achieved with the new strategy presented in the next section.
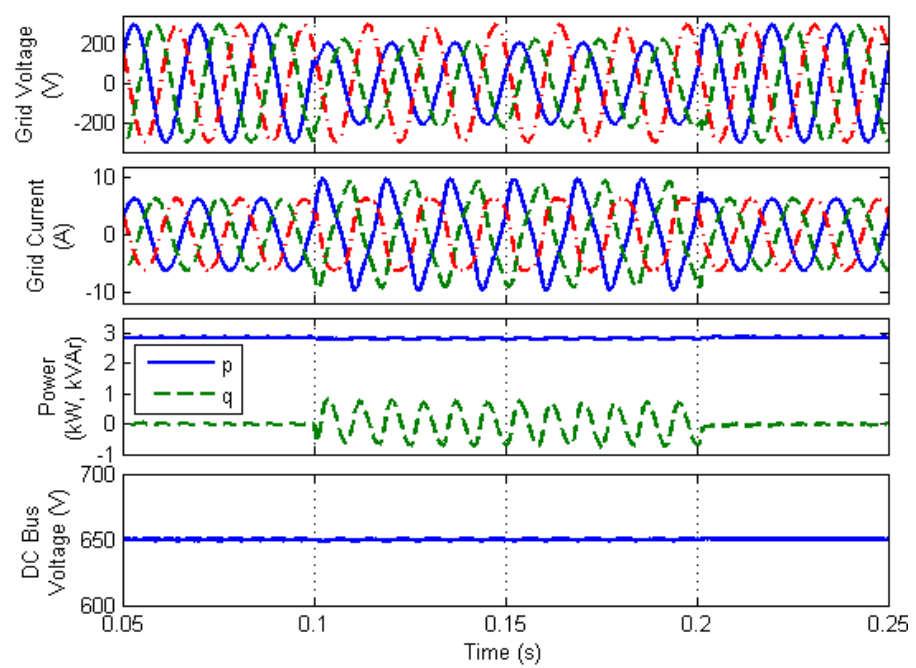

Fig. 2. Simulation results to classic PV control strategy under a phase-neutral fault. (a) Grid voltages, (b) Currents delivered by PV system, (c) Instantaneous active and reactive powers, (d) DC link voltage.

\section{PROPOSED CONTROL}

According to the instantaneous power theory, the instantaneous active power $p$ delivered by the inverter is given by

$$
p=\mathbf{v} \cdot \mathbf{i}
$$

where $\mathbf{v}=\left(v_{a}, v_{b}, v_{c}\right)$ is a voltage vector, $\mathbf{i}=\left(i_{a}, i_{b}, i_{c}\right)$ is a current vector and $\cdot$ represents the scalar product. For a given voltage vector, there are infinite current vectors, which are able to deliver exactly the same instantaneous active power to the grid. The current vector that results in sinusoidal currents is given by [7]:

$$
\mathbf{i}_{p}^{*}=\frac{P}{\left|\mathbf{v}^{+}\right|^{2}} \mathbf{v}^{+},
$$

where $P$ is the average active power and $\mathbf{v}^{+}$is the positivesequence voltage vector. The current vector in (2) consists of a set of perfectly balanced positive-sequence sinusoidal waveforms monotonously proportional to the positivesequence voltage vector. The DC voltage control exhibits slow dynamic, and hence, the active power reference will be considered a constant throughout each grid-voltage cycle, besides the vector $\mathbf{v}^{+}$has constant amplitude. Therefore the reference current vector will have constant amplitude. This amplitude is given by

$$
\hat{I}=\frac{2}{3} \frac{P}{\hat{V}^{+}},
$$

where the symbol $\wedge$ denotes peak value. From (3) it is possible to define the maximum power,

$$
P_{\text {LIMIT }}=\frac{3}{2} \hat{I} \hat{V}^{+},
$$

that can be delivered to the grid keeping the current amplitude, $\hat{I}$, beside the inverter limits. Note that the limiter is a function of positive sequence voltage, so any fault that results in a decrease of this value will reduce the average power delivered by the inverter.

The development above is made on generic coordinate system and then can be used in the stationary reference frame as well as in any other.

\section{A. Implementation of proposed control}

The overall block diagram of the proposed control is shown in Fig. 3. The error of square DC voltage is applied to a PI controller resulting in an active power reference. The photovoltaic power generation is added to this reference to give the inverter active power reference. A notching filter tuned at twice fundamental frequency is used to disregard the DC bus oscillation that occurs under fault. The saturation limit of the power reference is not hard and must change in function of positive sequence voltage, as suggested in (4), to keep the current limit. To calculate the power limit it is necessary to get the grid positive-sequence voltage, besides the knowledge of its angular frequency to perform the current control. This information about the grid voltage and frequency can be obtained through a Frequency-Locked Loop (FLL) proposed in [8]. It is a simple way to get the positive alpha beta components of the grid voltage and the system frequency even if the grid voltages are unbalanced.

The current control is made on stationary reference frame using a PR controller to assuring zero error on steady-state. The output of the PR controller is added to grid voltage, $v_{\alpha \beta}$, to perform a feed-forward control loop. 


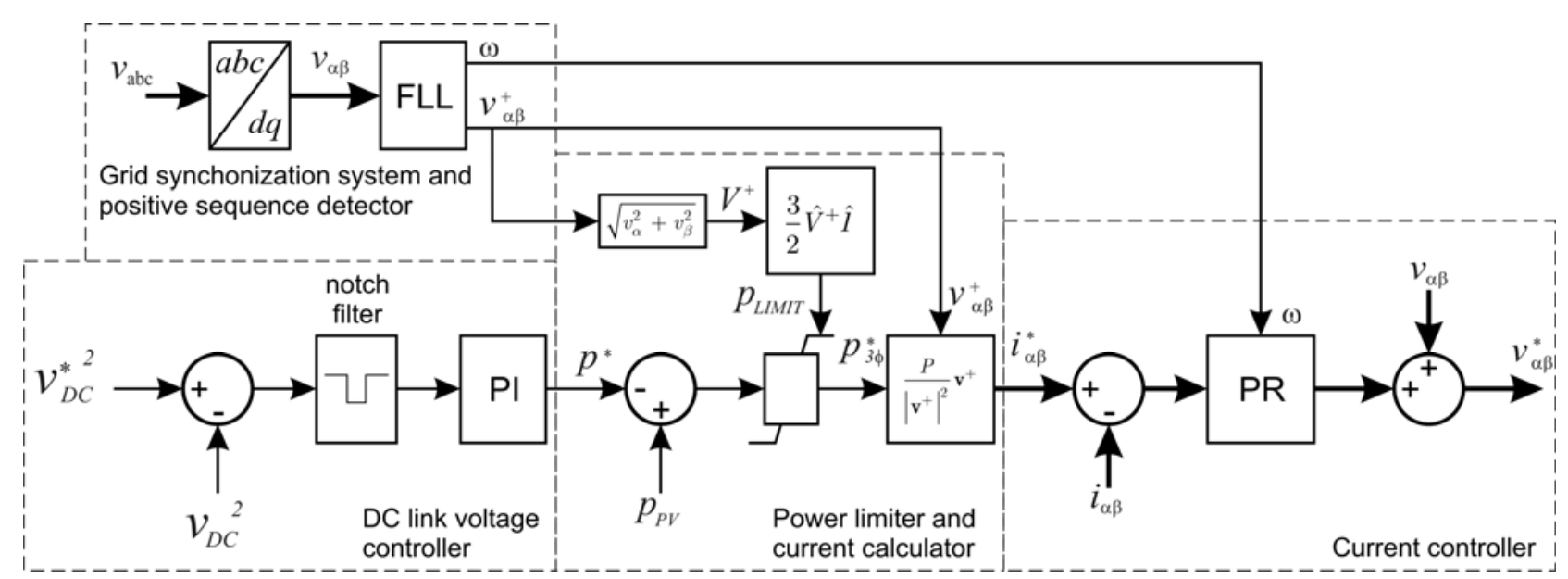

Fig. 3. Overall control diagram of the proposed controller and its sub-blocks.

\section{B. Simulation results}

The performance of the proposed control has been tested by means of simulations carried out in PSCAD/EMTDC, where a $3 \mathrm{~kW}$ photovoltaic system is supposed to be connected to a $380 \mathrm{~V}$ grid at the PCC. The PV array model has an open-circuit voltage of $832 \mathrm{~V}$ and the short-circuit current of $5.28 \mathrm{~A}$ at $S=1000 \mathrm{~W} / \mathrm{m}^{2}$ and $T=40^{\circ} \mathrm{C}$. The grid is modeled by a source connected to the transformer $T_{1}$ through two lines that are represented by their equivalent impedances, $Z_{L 1}$ and $Z_{L 2}$. The whole system is shows in Fig. 4.

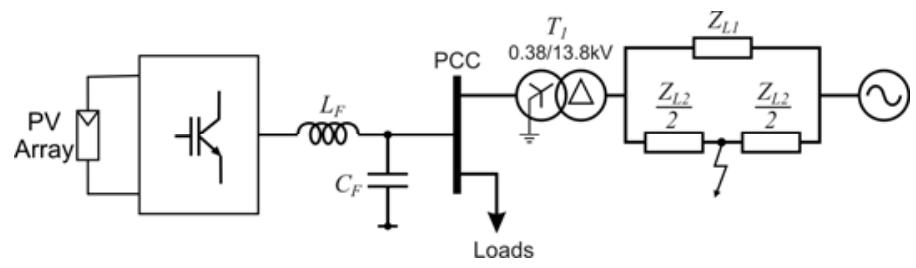

Fig. 4. Model of the system used in simulation.

In order to analyze the behaviour of the presented strategy in a faulty scenario a phase to ground fault will be applied at midpoint of the line 2, giving rise to a voltage sag at this node. The voltages at PCC in this time are show in Fig. 5a. The positive-sequence voltage at PCC before the fault is $212 \mathrm{~V}_{\mathrm{RMS}}$ and throughout the fault is $170 \mathrm{~V}_{\mathrm{RMS}}$. It was assumed a hypothetic condition that the inverter was delivering rated power, with rated current, when the fault occurs. The current limit was set to the same value of rated current (6.5 A of peak). Therefore can be noted that the current peak value do not change even under the fault as shown in Fig. 5b. Moreover, the currents are sinusoidal and balanced. Figure 5c shows the active and reactive power delivered by de PV system. Throughout the fault these powers are oscillating due the interaction between the negative sequence of the voltage and the positive sequence of the current. Note that the average value of the reactive power is zero as desired, but the average value of the active power is less than before the fault. It occurs because the positive-sequence of the voltage decreases too and the control decreases this power to do not exceed the rated current. Whereas the output power decrease the generated PV power tend to remain constant, so the DC bus voltage become to increase, as shown in Fig. 5d, and consequently the PV power goes down due the output characteristic of the PV cell. The DC voltage increase is not a problem because it never reaches the PV open circuit voltage.

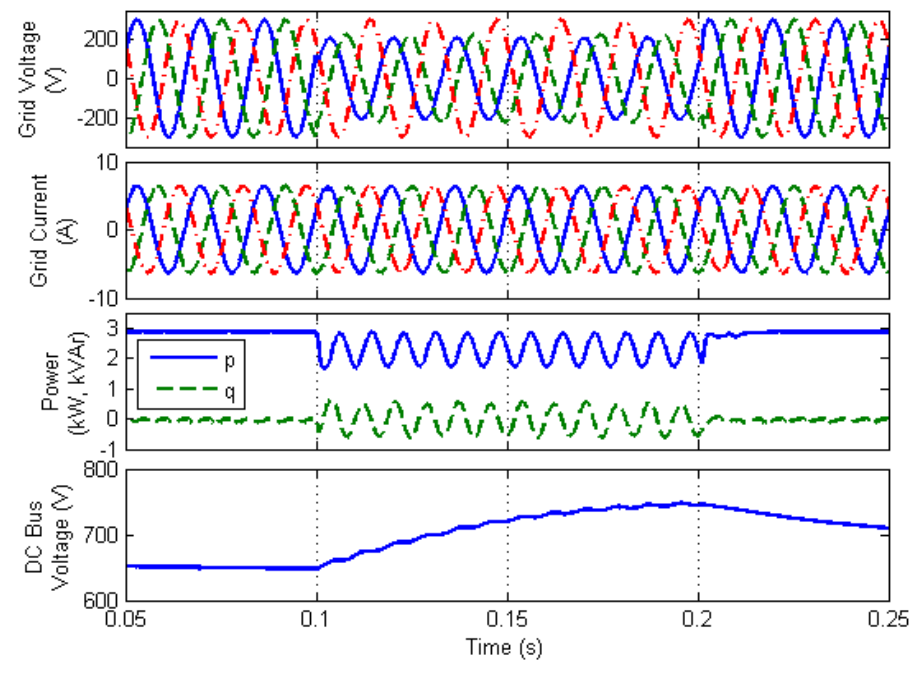

Fig. 5. Performance of the proposed control system under an asymmetric fault when it has delivering nominal current. (a) Grid voltages, (b) Currents delivered by the PV system, (c) Instantaneous active and reactive powers, (d) DC link voltage.

The same simulation is repeated assuming that the power generated by the PV array is lower. The results to this case are shown in Fig. 6. The power delivered to the grid is constant and equal to $1.58 \mathrm{~kW}$ before the fault. It becomes oscillatory during the fault but its average value is the same. In this case the active power does not fall because the inverter is able to supply this power without reaching the rated current. Thus the current amplitude increases a little bit as an action to the reduction in the positive-sequence voltage. 


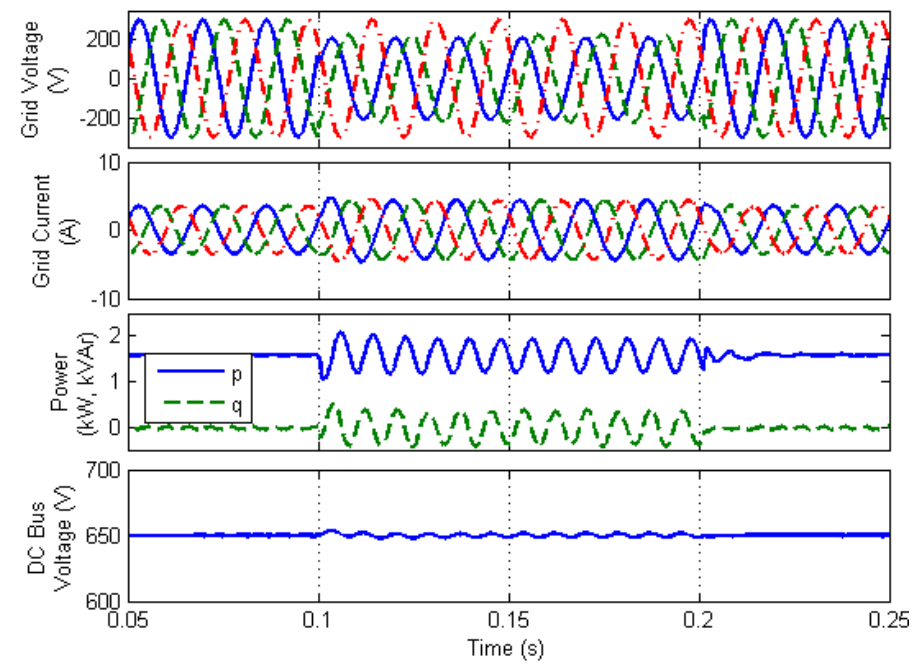

Fig. 6. Performance of the proposed control system under an asymmetric fault when it has delivering lowest power. (a) Grid voltages, (b) Currents delivered by the PV system, (c) Instantaneous active and reactive powers, (d) DC link voltage.

\section{EXPERIMENTAL RESULTS}

The control strategies have been implemented in an experimental setup, similar to Fig. 4, comprising a currentcontrolled VSI and an LC filter connected to the grid through a $\Delta y$ transformer. Moreover the grid consists of another transformer with taps. The fault was generated switching the tap of the grid transformer giving rise to voltage sag of $50 \%$ on phase $c$. This single-phase fault is propagated to the $y$ winding of the transformer gives rise to a phase-phase fault. The voltage

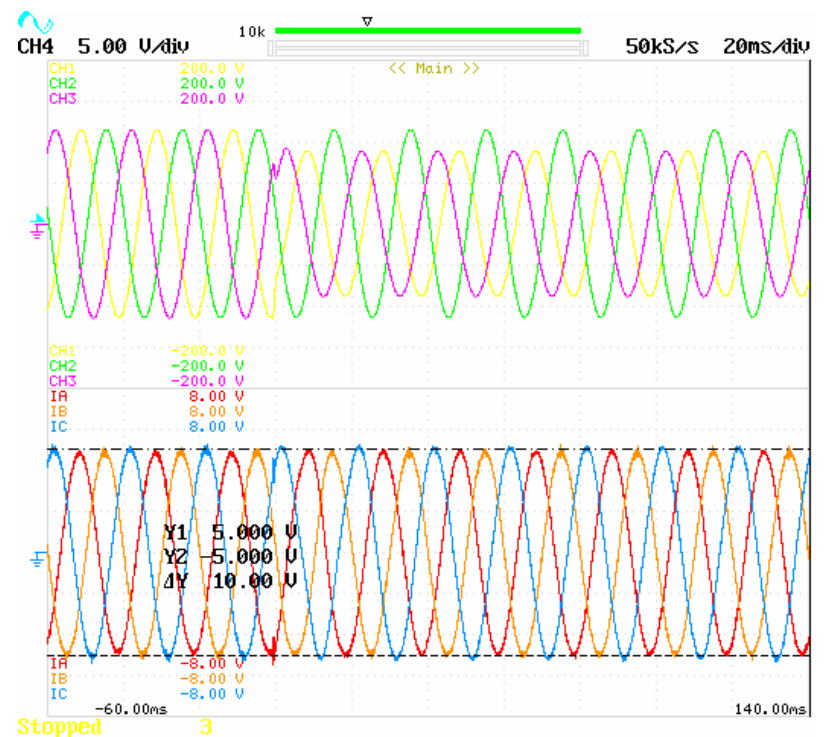

(a) at PCC was 70 Vrms before the fault. The PV array was changed by a DC-power source, which was set to $300 \mathrm{~V}$, in order to evaluate only the current limiter strategy. A dSpace 1103 DSP card is used to implement the current limiter controller, the FLL for detecting positive- and negativesequence voltage components [8], and the algorithm to calculate the current references on synchronous reference frame. The sampling frequency is set to $10 \mathrm{kHz}$ and switching frequency is set to $20 \mathrm{kHz}$.

The Fig. 7a shows the voltage at PCC and the currents injected by the inverter. The current scale is of $1 \mathrm{~A} / \mathrm{V}$. The power delivered by the inverter was set to resulting in a current peak of $5 \mathrm{~A}$ and the current limit, $\hat{I}$, was set to the same value, so when the fault occurs the current is kept under this limit value. The active and reactive powers for this experiment are show in Fig. 7b. Note that throughout the fault the active power is oscillating and its average value is $730.6 \mathrm{~W}$ whereas before it was $870.2 \mathrm{~W}$. It occurs due the decreased in the positivesequence voltage. Consequently to keep the current peak the average active power must be lower, as suggest in (4).

The experiment was repeated with the current limiter inactive through the first $60 \mathrm{~ms}$ after the fault to evaluate the effectiveness of the strategy. When the dip occurs the current peak increase around $16 \%$ because the current limiter was inactive and return to $5 \mathrm{~A}$ just after enable it, as shown in Fig. 8a. The Fig. $8 \mathrm{~b}$ shows that the average active power through the $60 \mathrm{~ms}$ after the dip is the same as before resulting in overcurrent. The value of overcurrent is proportional to the sag in the positive-sequence voltage. Thus for most severe faults the overcurrent reaches higher values than that shown here.

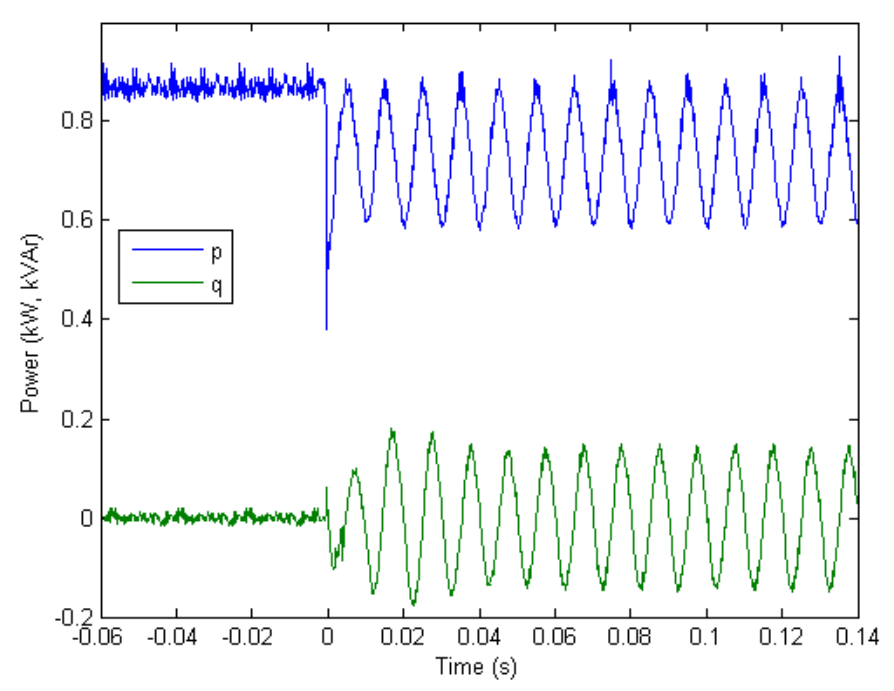

(b)

Fig. 7. Experimental results of the proposed control system towards a dip type C. (a) Grid voltages and currents, (b) Active and reactive power delivered to the grid. 


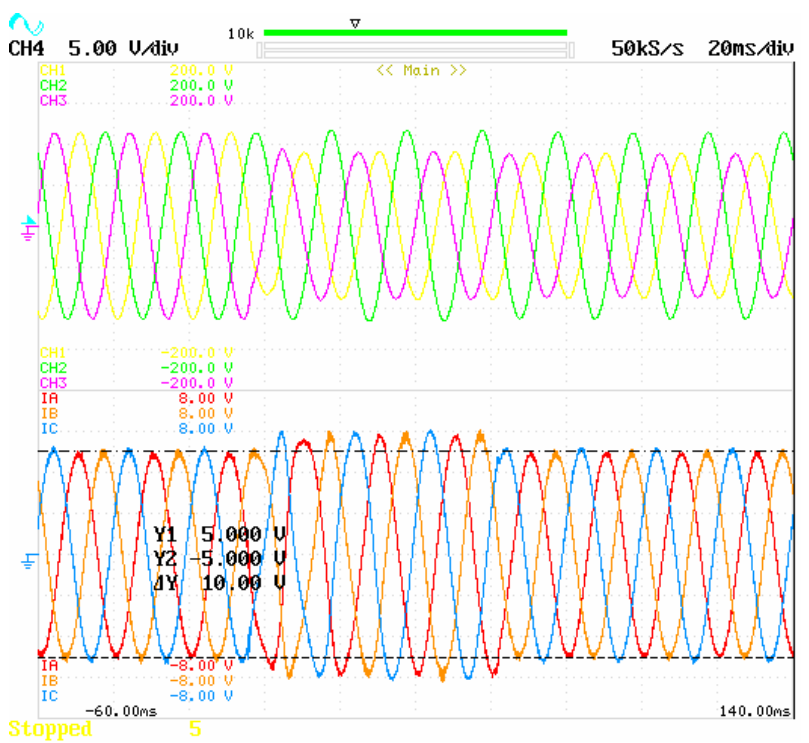

(a)

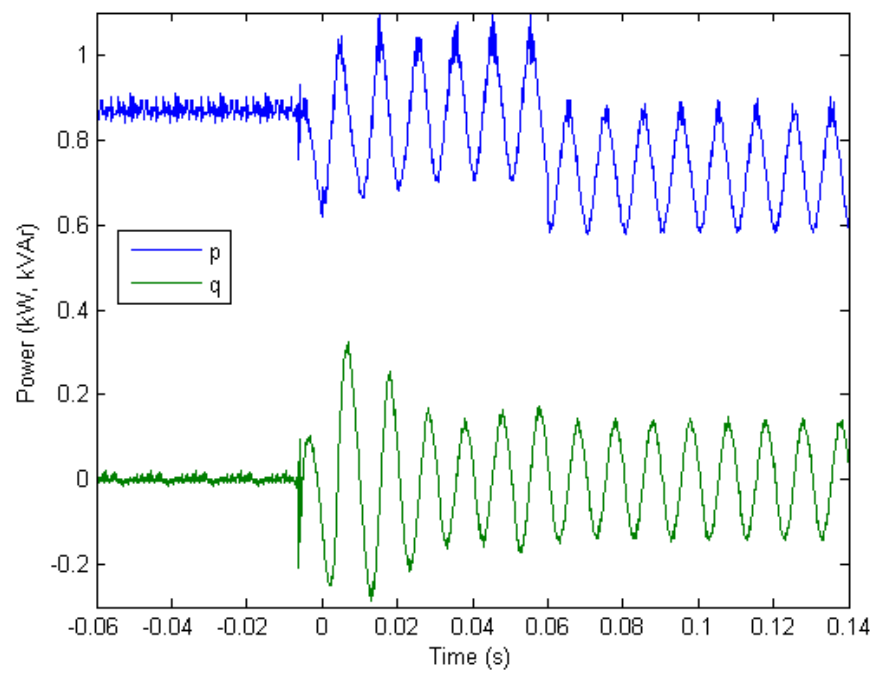

(b)

Fig. 8. Experimental results of the control system towards a dip type $\mathrm{C}$ with the control limit disabled in the first $60 \mathrm{~ms}$ after the fault, and then it is turn on. (a) Grid voltages and currents, (b) Active and reactive power delivered to the grid.

\section{CONClusions}

This paper proposes a control strategy for three-phase PV systems connected to the electrical grid. The proposed control strategy permits the inverter operates in any fault situation without overcurrents as well as delivering sinusoidal currents. Its implementation is as simple as classic strategies and it can be applied to other DG systems as long as the power of the primary source can be limited. The effectiveness of the proposed control strategy is evaluated through simulation in PSCAD and experimental results.

\section{ACKNOWLEDGMENT}

The author would like to thank CAPES, CNPq and the Spanish Ministry of Education and Science (grant PHB 200900?-PC) for their financial support.

\section{REFERENCES}

[1] VDE 0126-1-1, Automatic disconnection device between a generator and the public low-voltage grid

[2] B. Bletterie, R. Bründlinger, H. Fechner; "Sensitivity of photovoltaic inverters to voltage sags - test results for a set of commercial products;" 18. International Conference on Electricity Distriution, 2005, Turin, Italien

[3] Teodorescu, R.; Blaabjerg, F.; Liserre, M.; Loh, P.C., "Proportionalresonant controllers and filters for grid-connected voltage-source converters," Electric Power Applications, IEE Proceedings - , vol.153, no.5, pp.750-762, September 2006

[4] Esram, T.; Chapman, P.L., "Comparison of Photovoltaic Array Maximum Power Point Tracking Techniques," Energy conversion, IEEE transactions on, vol.22, no.2, pp.439-449, June 2007

[5] Cavalcanti, M. C.; Azevedo, G. M. S.; Amaral, B. A.; Oliveira, K. C.; Neves, F A. S.; Lins, Z. D., "A Grid Connected Photovoltaic Generation System with Harmonic and Reactive Power Compensation," In: Congresso Brasileiro de Eletronica de Potencia, 2005, Recife. COBEP2005. Recife, 2005.
[6] Schonardie, M.F.; Martins, D.C., "Grid-connected Photovoltaic Threephase System Using Park Transformation With Active And Reactive Power Control And Input Voltage Clamped," In: Congresso Brasileiro de Eletronica de Potencia, 2007, Blumenau. COBEP2007.

[7] Komatsu, Y., "Application of the extension pq theory to a mains-coupled photovoltaic system," Power Conversion Conference, 2002. PCC Osaka 2002. Proceedings of the , vol.2, no., pp.816-821 vol.2, 2002

[8] Rodriguez, P.; Timbus, A.V.; Teodorescu, R..; Liserre, M..; Blaabjerg, F.., "Flexible Active Power Control of Distributed Power Generation Systems During Grid Faults," Industrial Electronics, IEEE Transactions on , vol.54, no.5, pp.2583-2592, Oct. 2007.

[9] Rodriguez, P.; Luna, A.; Ciobotaru, M.; Teodorescu, R.; Blaabjerg, F., "Advanced Grid Synchronization System for Power Converters under Unbalanced and Distorted Operating Conditions," IEEE Industrial Electronics, IECON 2006 - 32nd Annual Conference on , vol., no., pp.5173-5178, 6-10 Nov. 2006 